\title{
Rasgos de personalidad y dificultades de regulación emocional en estudiantes universitarios
}

\author{
Macarena del Valle ${ }^{1}$, Eliana Vanesa Zamora ${ }^{2}$, Yasmin Khalil ${ }^{3}$ y Macarena Altamirano ${ }^{4}$
}

\begin{abstract}
RESUMEN
La personalidad constituye uno de los principales constructos psicológicos debido a su poder explicativo y predictivo sobre el comportamiento humano. Recientemente, muchos estudios se han focalizado en la relación entre la personalidad y la regulación y control de las emociones. Por este motivo, el presente estudio se propuso indagar acerca de las relaciones entre los rasgos de personalidad del Modelo de los Cinco Grandes Factores y las dificultades que las personas pueden tener al momento de regular sus emociones. Se trabajó con un total de 195 participantes, estudiantes regulares de la Universidad Nacional de Mar del Plata ( $84.1 \%$ mujeres, $15.9 \%$ hombres), con edades comprendidas entre los 18 y los 35 años $(M=22.85 ; D E=4.22)$. Los estudiantes que accedieron a participar voluntariamente del estudio completaron la adaptación de la Escala de Dificultades en la Regulación Emocional y el listado de Adjetivos para Evaluar la Personalidad. Los resultados indicaron que el rasgo con mayor efecto explicativo sobre la desregulación emocional fue el Neuroticismo. Se observó que mientras mayor es la presencia del rasgo, mayor es la presencia de dificultades de regulación emocional. Asimismo, los rasgos Amabilidad y Extraversión también aportaron a la varianza explicada, aunque de forma menor. Los rasgos Responsabilidad y Apertura a la Experiencia no resultaron significativos en el modelo de regresión para las dificultades de regulación emocional. Este estudio supone un aporte al esclarecimiento de las relaciones entre la personalidad y los mecanismos de regulación emocional, específicamente en población estudiantil universitaria.

Palabras Clave: regulación emocional, personalidad, neuroticismo, afabilidad, estudiantes universitarios, rasgos de personalidad, Modelo de los Cincos Grandes Factores.
\end{abstract}

\section{Personality traits and emotion dysregulation in university students}

\begin{abstract}
Personality is one of the main psychological constructs due to its explanatory and predictive power over human behavior. Recently many studies have focused on the relationship between personality and the regulation and control of emotions. For this reason, the present study examines the relationships between the five personality traits of the Big Five Factor Model and the difficulties that people may experience when regulating their emotions. A sample of 195 college students from the National University of Mar del Plata was used $(84.1 \%$ women, $15.9 \%$ men). Ages ranged from 18 to 35 years old $(M=$ 22.85; $S D=4.22$ ). Those students who voluntarily agreed to participate in the study

${ }^{1}$ Consejo Nacional de Investigaciones Científicas y Técnicas (CONICET), Universidad Nacional de Mar del Plata, Instituto de Psicologia Básica, Aplicada y Tecnología (IPSIBAT), Argentina;

mdelvalle1989@gmail.com; https://orcid.org/0000-0003-3549-7224

2 Consejo Nacional de Investigaciones Científicas y Técnicas (CONICET), Instituto de Psicología Básica,

Aplicada y Tecnologia(IPSIBAT), Argentina; elianavanesazamora@gmail.com; https://orcid.org/00000002-6278-6665

3 Universidad Nacional de Mar del Plata, Argentina; yaskha2@gmail.com; https://orcid.org/0000-00018805-7367

${ }^{4}$ Universidad Nacional de Mar del Plata, Argentina; macaaltamirano8@gmail.com; https://orcid.org/00000002-1612-8354
\end{abstract}


completed the adaptation of the Difficulties in Emotional Regulation Scale and the Adjectives Checklist for Personality Assessment. Results indicated that the trait with the greatest explanatory effect on emotional dysregulation was Neuroticism: the greater the presence of the trait, the greater the presence of difficulties in emotion regulation. Likewise, Agreeableness and Extraversion traits also contributed to the variance, although in a smaller way. Conscientiousness and Openness to Experience traits resulted not significant in the regression model for emotion dysregulation. The study is a contribution to the clarification of the relationships between personality and the mechanisms of emotional regulation and dysregulation in college student's population.

Keywords: emotion regulation, personality, neuroticism, extraversion, agreeableness, college students, personality traits, Big Five Factor Model.

La personalidad constituye uno de los principales constructos psicológicos debido a su poder explicativo y predictivo sobre el comportamiento humano (Aizen, 2005). Recientemente, diversos estudios han sugerido (e.g., Andrés, 2014; Pollock, McCabe, Southard, \& Zeigler-Hill, 2016; Purnamaningsih, 2017) que los rasgos constitutivos de la personalidad podrian guardar relación con la forma en la que las personas regulan sus emociones. La personalidad puede ser definida como el patrón relativamente estable de pensamiento, sentimiento y actuación que caracteriza a una persona (Costa \& McCrae, 1985). Actualmente, se considera que la personalidad se encuentra conformada por unidades más básicas, denominadas rasgos, los cuales, según la propuesta de Allport (1937), son disposiciones estables y consistentes, que generan patrones de comportamiento (Romero, 2005).

Se concibe a la personalidad como un constructo multidimensional, propuesta que tiene base en los aportes seminales realizados por Cattell (1946) y Eysenck (Eysenck \& Eysenck, 1985), y desarrollados más tarde por Costa y McCrae (1985). Estos autores entienden a la personalidad como una unidad compuesta por rasgos fundamentales que permiten caracterizar y predecir el comportamiento humano. Mientras que la propuesta de Cattell consideraba la existencia de 16 factores y Eysenck sugería tres, el modelo de Costa y McCrae propone la existencia de cinco factores y es llamado Modelo de los Cinco Grandes Factores (Big Five Factor Model). En este modelo, los autores postulan que estos rasgos poseen base genética, estabilidad temporal, estructura universal, y no dependen de la influencia del ambiente. Los cinco factores poseen características distintivas y se expresan, cada uno, en dos polos de un continuo: Neuroticismo-Estabilidad emocional, ExtraversiónIntraversión, Apertura a la experiencia-Cerrado a la experiencia, AmabilidadHostilidad y Responsabilidad-Irresponsabilidad. A lo largo de los años, diferentes estudios han aportado evidencias que confirman la estructura pentafactorial de la personalidad (e.g., Allik, Realo, \& McCrae, 2013; Goldberg, 1990; McCrae \& Costa, 1987).

Los rasgos de personalidad permiten predecir conductas de diverso tipo como el uso de sustancias (Kotov et al., 2010), el desempeño académico (Cupani, Garrido, \& Tavella, 2013), el bienestar psicológico (Garaigordobil, Aliri, \& Fontaneda, 2009) o la calidad de vida (Huang et al., 2017). Si bien son numerosas y diversas las investigaciones que abordan la personalidad, el análisis de su relación con la regulación emocional ha sido estudiado en menor proporción (Andrés, 2014). 
La regulación emocional puede ser definida como el conjunto de procesos mediante los cuales las personas expresan, monitorean y modifican sus emociones en función del contexto (Gross, 1999). Gratz y Roemer (2004) sugieren un modelo clínico de la regulación emocional basado en seis dificultades que pueden presentar las personas al regular sus emociones negativas: falta de aceptación emocional, dificultad para orientar el comportamiento dirigido a metas, dificultad en el control de los impulsos, falta de reconocimiento y conciencia emocional, acceso limitado a las estrategias de regulación emocional y falta de claridad emocional. Para evaluar estas dimensiones, los autores diseñaron la Escala de Dificultades de Regulación Emocional, la cual es en la actualidad ampliamente utilizada en población clínica y no clínica de distintos países (e.g., Côté, Gosselin, \& Dagenais, 2013; Guzmán-González, Trabucco, Urzúa, Garrido, \& Leiva, 2014; Pollock et al., 2016). No obstante, la estructura factorial del constructo de desregulación emocional es aún materia de debate (ver Rodríguez-Camejo, 2019). En un estudio reciente realizado en el ámbito local (Medrano \& Trógolo, 2016), se puso a prueba el modelo de Gratz y Roemer, hallando que una solución de dos factores presentaba un mejor ajuste que el modelo original de seis factores. Estas dos dimensiones halladas referirian a las Dificultades en el procesamiento emocional y a las Dificultades en la regulación de la respuesta emocional. Mientras que la primera refiere a las dificultades para reconocer, identificar y aceptar las emociones (incluyendo la falta de aceptación emocional y la falta de claridad emocional), la segunda engloba a las dificultades para controlar los impulsos derivados de las respuestas emocionales y a la capacidad de regular o gestionar esas respuestas para el logro de objetivos personales (incluyendo la dificultad para orientar el comportamiento dirigido a metas y la dificultad en el control de los impulsos).

Ahora bien, mientras que la adecuada regulación de las emociones está asociada al bienestar emocional, psicológico y físico del individuo (e.g., Mateo, Faixa, \& Martín-Asuero, 2014), la presencia de dificultades para regular las emociones se encuentra asociada a distintos trastornos psicopatológicos (e.g., McLaughlin, Hatzenbuehler, Mennin, \& Nolen-Hoeksema, 2011; Pérez Díaz \& Guerra Morales, 2014; Hervás, 2011) y resulta desadaptativa, disfuncional y negativa para el individuo y su ajuste emocional (Hervás \& Moral, 2017). Es posible que las diferencias interindividuales que se observan en la vida adulta en la forma de regular las emociones se deban a variaciones en las dimensiones de la personalidad (John \& Gross, 2004), especialmente en los rasgos Neuroticismo y Extraversión (Andrés, Canet Juric, Castañeiras, \& Richaud de Minzi, 2016). Por ejemplo, ciertos estudios reportan que el rasgo Neuroticismo se encuentra asociado a una mayor tendencia a experimentar emociones negativas y a regular las mismas de forma desadaptativa (e.g., Andrés, Castañeiras, \& Richaud, 2014; Austin, Dore, \& O’Donovan, 2008; Matsumoto, 2006; Pollock et al., 2016). De manera inversa, se han reportado asociaciones entre el rasgo Extraversión y la experimentación y expresión de emociones positivas (e.g., Diener, Oishi, \& Lucas, 2003). Además, las facetas que componen dicho rasgo, a saber, cordialidad, gregarismo, asertividad, actividad, búsqueda de emociones y emociones positivas, expresan aspectos importantes de la regulación emocional, tal como fue planteado por Gratz y Roemer (2004).

También, las personas que presentan altos niveles de Extraversión suelen ser más reactivas a estímulos agradables y a experimentar emociones positivas, mientras que aquellas que presentan altos niveles de Neuroticismo 
suelen experimentar emociones desagradables y poseen mayor reactividad a estímulos negativos (Andrés et al., 2014; Augusto Landa, Pulido Martos, \& López-Zafra, 2010; Rusting \& Larsen, 1998). El rasgo Neuroticismo permitiría predecir la desregulación emocional ante situaciones problemáticas ya que los sujetos con marcada presencia de este rasgo presentan reacciones negativas más perdurables y sostenidas en el tiempo, así como respuestas rumiativas y un estilo de afrontamiento desadaptativo (Carver, \& Connor-Smith, 2010; McLaughlin et al., 2011; Tong, 2010). Del mismo modo, se ha demostrado que las dimensiones Neuroticismo y Extraversión presentan diferencias en los modos de regular las emociones incluso en relación con la atención y claridad de estas (Coffey, Berenbaum, \& Kerns, 2003): la Extraversión correlaciona con una mayor capacidad de atención emocional, mientras que el Neuroticismo se asocia a una incapacidad de percibir con claridad los estados emocionales (Ramos, Morán, Pérez, \& Manga, 2007). En función de lo anteriormente planteado, los individuos con mayor Extraversión presentan mayores habilidades de regulación emocional y se encuentran más atentos a lo que sienten y expresan, mientras que en individuos con mayor Neuroticismo se observan menores habilidades de regulación emocional (Austin et al., 2008).

De forma similar, aunque menos claro en la literatura, resulta posible plantear que los rasgos Amabilidad, Apertura a la experiencia y Responsabilidad también guarden relación con las dificultades para regular las emociones. Por ejemplo, la Amabilidad y la Responsabilidad podrían asociarse con la emocionalidad positiva (McCrae \& Costa, 1991) y con la forma en la que las personas manejan sus emociones, las expresan y las comunican a los demás (McCrae \& Costa, 1997; Ramos et al., 2007; Reisenzein \& Weber, 2009; Tobin, Graziano, Vanman, \& Tassinary, 2000). Por su parte, la Apertura a la experiencia podría tener relación con el reconocimiento y aceptación de las propias emociones (e.g., Terracciano, Merritt, Zonderman, \& Evans, 2003).

Por último, es importante destacar que la mayoría de los autores acuerdan en que las emociones presentan aspectos adaptativos (Lauretti, Villalobos, \& González, 2007) y resultan facilitadoras de las respuestas adaptativas del organismo al ambiente (Mauss, Levenson, McCarter, Wilhelm, $\&$ Gross, 2005). Así, la capacidad para regular las emociones cumple un papel fundamental en la adaptación académica de los estudiantes, e influye sobre su rendimiento académico (Graziano, Reavis, Keane, \& Calkins, 2007; Gumora \& Arsenio, 2002). Actualmente, los resultados de estudios indican que la población de estudiantes universitarios es propensa a presentar trastornos emocionales y malestar en general (Erazo Caicedo, \& Jiménez Ruiz, 2012; Vélez, Gutiérrez, \& Isaza, 2010), lo cual puede afectar su rendimiento académico (Mega, Ronconi, \& De Beni, 2014). En consecuencia, la indagación sobre aquellos factores que pueden afectar los procesos emocionales en población universitaria reviste especial interés. A pesar de ello, son escasos los estudios en habla hispana que han abordado la relación entre personalidad y regulación emocional en el ámbito académico, y aún menos explorada ha sido esta relación en el nivel universitario. Por lo tanto, el presente estudio se propuso analizar la incidencia de los rasgos de personalidad sobre las dificultades de regulación emocional en población de estudiantes de nivel superior. 


\section{MÉTODO}

\section{Participantes}

Se trabajó con un total de 195 participantes, estudiantes regulares de la Universidad Nacional de Mar del Plata. Del total, $84.1 \%$ fueron mujeres ( $n=$ 164) y $15.9 \%$ fueron hombres $(n=31)$, lo cual resulta acorde con la proporción entre hombres y mujeres que siguen muchas carreras de dicha universidad. La edad media de los participantes fue de 22.85 años $(D E=4.22$; $\operatorname{Min}=18$; Máx = 35).

\section{Instrumentos}

Personalidad. Para evaluar los cinco rasgos de personalidad del modelo de los Cinco Grandes, se administró el listado de Adjetivos para Evaluar la Personalidad (AEP, Ledesma, Sánchez, \& Díaz-Lázaro 2011). Se trata de un listado de 67 adjetivos que reflejan los cinco aspectos de la personalidad del individuo y que se responden mediante una escala de tipo Likert, con cinco opciones de respuesta $(1=$ no me describe en absoluto, $5=$ me describe tal como soy). El instrumento presenta valores de confiabilidad Alpha de Cronbach (a) adecuados (Tavakol \& Dennick, 2011) para las dimensiones de Extraversión $(a=.79)$ y Apertura a la Experiencia $(a=.74)$ y muy buenos para las dimensiones de Amabilidad $(a=.84)$, Neuroticismo $(a=.85) \mathrm{y}$ Responsabilidad $(a=.80)$ (Ledesma et al., 2011). En este estudio, los indices de confiabilidad resultaron adecuados para las dimensiones de Apertura a la Experiencia $(a=.71)$ y Responsabilidad $(a=.79)$ y muy buenos para las dimensiones de Amabilidad $(a=.82)$, Neuroticismo $(a=.84)$ y Extraversión: $a$ $=.82)$.

Dificultades de regulación emocional. Para evaluar la frecuencia de dificultades en la regulación emocional, se aplicó la adaptación argentina (Medrano \& Trógolo, 2014, 2016) de la Escala de Dificultades en la Regulación Emocional (DERS) originalmente desarrollada por Gratz y Roemer (2004). La escala original incluye 36 items con un formato de respuesta de tipo Likert de cinco puntos ( $1=$ casi nunca, $5=$ casi siempre $)$, los cuales se dividen en seis factores que representan distintas dificultades que las personas pueden presentar a la hora de regular sus emociones (Falta de aceptación emocional, Interferencia en conductas dirigidas a metas, Dificultades en el control de impulsos, Falta de conciencia emocional, Falta de claridad emocional, y Acceso Limitado a Estrategias de Regulación Emocional). En cambio, la adaptación realizada por Medrano y Trógolo $(2014,2016)$ retiene 21 items, que pueden agruparse en dos grandes factores de segundo orden, a saber, Dificultades en el procesamiento emocional y Dificultades en la regulación de la respuesta emocional. Mientras que la primera dimensión refiere a las dificultades para reconocer, identificar y aceptar las emociones, la segunda supone dificultades para controlar los impulsos derivados de las respuestas emocionales y ser capaz de regular o gestionar esas respuestas para el logro de objetivos personales. El modelo propuesto en la adaptación presentó adecuados índices de ajuste, siendo el CFI de .90 y el RMSEA de .07. La confiabilidad, evaluada mediante el $a$ de Cronbach, fue buena para ambas dimensiones del instrumento, siendo .82 el a de Procesamiento emocional y .88 el de Respuesta emocional. Los coeficientes de confiabilidad en el presente estudio fueron buenos tanto para la escala total, con un a de .90, como para 
las dimensiones de Procesamiento emocional y Respuesta emocional, con valores $a$ de .84 y .88 , respectivamente.

Los datos referidos a las variables sociodemográficas de género y edad fueron preguntados en el encabezado de los protocolos junto con el consentimiento informado.

\section{Procedimiento}

El presente estudio forma parte de un proyecto general aprobado por la Secretaría de Investigación de la Universidad Nacional de Mar del Plata. Los autores del estudio fueron los responsables de la administración de los instrumentos. Se aplicaron las escalas DERS y AEP en distintas clases prácticas y teóricas de la Universidad Nacional de Mar del Plata. Se contactó a los docentes responsables de forma previa al inicio de la clase y se solicitó su aprobación para que los alumnos participaran del estudio, indicando que en total el proceso demoraría aproximadamente 15 a 20 minutos. Se comentaron de manera grupal las características y objetivos del estudio y se permitió a los participantes hacer preguntas. Quienes accedieron a participar de forma voluntaria debieron firman un consentimiento informado que consistía en la información detallada de los objetivos del estudio, procedimientos y protección de datos personales. Además, se consignaba quiénes eran los responsables del estudio y la posibilidad de abandonar el mismo si así lo deseaban.

A continuación, se entregaron los protocolos correspondientes a ambas escalas en formato de papel y lápiz. El estudio siguió en todo momento los lineamientos para la conducta ética en investigación en seres humanos recomendados por la Asociación Americana de Psicología (American Psychological Association, 2010), así como los principios éticos de la Declaración de Helsinki (World Medical Association, 2013).

\section{Análisis de los datos}

Para el análisis de los datos, se trabajó con el Paquete Estadístico para Ciencias Sociales (SPSS) v. 23. Se analizó la confiabilidad de las escalas mediante el estadístico a de Cronbach. Para caracterizar la muestra, se realizaron análisis estadísticos descriptivos de las variables bajo estudio. Se calculó también una variable total de la escala DERS que representa la sumatoria de las dificultades de regulación emocional, tal como ya ha sido reportado por otros estudios (e.g., Giovannini et al., 2014; Karatzias et al., 2016). Para determinar la existencia de relaciones entre las variables bajo estudio, y dado que la muestra se encontraba desbalanceada en función del género, se aplicaron correlaciones parciales controlando el efecto de este último. Luego, para determinar la incidencia de los rasgos de personalidad sobre las dificultades de regulación emocional, se probaron tres modelos de regresión lineal mediante el método Entrar (Enter). En los tres modelos de regresión, las variables independientes introducidas fueron los cinco grandes rasgos de personalidad (Extraversión, Amabilidad, Neuroticismo, Responsabilidad y Apertura a la Experiencia) y el género. En el primer modelo, la variable dependiente fue la dimensión de dificultades en el Procesamiento emocional. En el segundo modelo, la variable dependiente fue la dimensión de dificultades en la regulación de la Respuesta emocional. Finalmente, para el tercer modelo, la variable dependiente fue el total de la escala DERS, es decir, la totalidad de las dificultades de regulación emocional. Los diagnósticos de colinealidad resultaron inferiores a 1.3 para todas las variables (VIF). Además, 
las variables dependientes, así como los residuos de los tres modelos, presentaron una distribución normal según lo indicado por la prueba de Kolmogorov-Smirnov $(p<.01)$.

\section{RESULTADOS}

Para analizar el grado de correlación entre las variables bajo estudio, se aplicaron análisis de correlación $r$ de Pearson. Los resultados se presentan en la tabla 1 junto con los valores estadísticos descriptivos.

Tabla 1.

Correlaciones parciales entre los rasgos de personalidad y las dificultades de regulación emocional controlando género.

\begin{tabular}{lcccccccccc}
\hline & $\boldsymbol{M}$ & $\boldsymbol{D E}$ & $\mathbf{1}$ & $\mathbf{2}$ & $\mathbf{3}$ & $\mathbf{4}$ & $\mathbf{5}$ & $\mathbf{6}$ & $\mathbf{7}$ & $\mathbf{8}$ \\
\hline 1. Extraversión & 3.66 & 0.67 & - & & & & & & \\
2. Amabilidad & 3.98 & 0.46 & $.30^{* *}$ & - & & & & & \\
3. Responsabilidad & 3.73 & 0.56 & $.19^{* *}$ & $.30^{* *}$ & - & & & & \\
4. Neuroticismo & 2.78 & 0.61 & $-.30^{* *}$ & $-.30^{* *}$ & $-.33^{* *}$ & - & & & \\
5. Apertura a la experiencia & 3.43 & 0.60 & $.31^{* *}$ & $.14^{*}$ & -.09 & -.10 & - & & \\
6. Procesamiento Emocional & 2.10 & 0.69 & $-.31^{* *}$ & $-.31^{* *}$ & $-.24^{* *}$ & $.60^{* *}$ & -.11 & - & \\
7. Respuesta Emocional & 2.23 & 0.74 & $-.32^{* *}$ & $-.33^{* *}$ & $-.27^{* *}$ & $.66^{* *}$ & -.09 & $.58^{* *}$ & - & \\
8. DERS Total & 2.17 & 0.64 & $-.35^{* *}$ & $-.36^{* *}$ & $-.28^{* *}$ & $.71^{* *}$ & -.11 & $.88^{* *}$ & $.90^{* *}$ & - \\
\hline
\end{tabular}

Nota: ${ }^{* *} p<.01,{ }^{*} p<.05$

Puede observarse que el rasgo de personalidad que presentó mayores asociaciones fue Neuroticismo. Las mismas resultaron directas y fuertes, indicando que mientras mayor es la presencia de dicho rasgo, mayor es la frecuencia de aparición de dificultades de regulación emocional, tanto para el procesamiento y reconocimiento de las emociones como para la gestión de la reacción emocional. A su vez, los rasgos Extraversión, Amabilidad y Responsabilidad también presentaron asociaciones con las dimensiones de regulación emocional, aunque las mismas fueron bajas e inversas. El rasgo Apertura a la experiencia no mostró relaciones estadísticamente significativas.

A continuación, para determinar la proporción de varianza explicada por cada uno de los rasgos de personalidad sobre las dificultades de regulación emocional, se probaron tres modelos de regresión mediante el análisis de regresión lineal múltiple (método Entrar). Las variables dependientes fueron respectivamente: 1) la dimensión de dificultades en el Procesamiento emocional; 2) la dimensión de dificultades en la regulación de la Respuesta emocional; 3) el total de la escala DERS, es decir, la totalidad de las dificultades de regulación emocional. En los tres casos, las variables independientes fueron los cinco rasgos de personalidad. Los tres modelos resultaron estadísticamente significativos. Los resultados se muestran en la tabla 2. 
Tabla 2.

Modelos de regresión del efecto de los rasgos de personalidad sobre las dificultades de regulación emocional.

\begin{tabular}{lccc}
\hline & $\begin{array}{c}\text { Procesamiento } \\
\text { emocional }\end{array}$ & $\begin{array}{c}\text { Respuesta } \\
\text { emocional }\end{array}$ & $\begin{array}{c}\text { DERS } \\
\text { Total }\end{array}$ \\
\cline { 2 - 4 } & $\boldsymbol{\beta}$ & $\boldsymbol{\beta}$ & $\boldsymbol{\beta}$ \\
\hline 1. Extraversión & -.11 & -.11 & $-.13^{*}$ \\
2. Amabilidad & -.11 & $-.12^{* *}$ & $-.13^{*}$ \\
3. Responsabilidad & -.01 & -.01 & -.01 \\
4. Neuroticismo & $.54^{* \star}$ & $.60^{* *}$ & $.64^{* *}$ \\
5. Apertura a la experiencia & -.01 & .02 & .01 \\
6. Género & .02 & .09 & .06 \\
\hline$r^{2}$ & .40 & .47 & .55 \\
$F_{(6,188)}$ & $20.44^{* *}$ & $27.82^{* *}$ & $37.93^{* *}$ \\
\hline
\end{tabular}

Nota: ${ }^{*} p<.05,{ }^{* *} p<.01 ; \beta$ : beta estandarizados.

Puede observarse que la varianza explicada es mayor para las dificultades en la regulación de la Respuesta emocional que para las dificultades en el Procesamiento emocional. El rasgo Neuroticismo resultó el mayor predictor en los tres modelos. ambién los rasgos Extraversión y Amabilidad aportaron a la varianza explicada, aunque con una proporción mucho menor: Extraversión solo sobre la escala total y Amabilidad sobre la escala total y sobre la dimensión de dificultades en la Respuesta emocional. En cambio, los rasgos Responsabilidad y Apertura a la experiencia no resultaron predictores en ningún modelo.

\section{DISCUSIÓN}

Este estudio indagó en las relaciones entre los rasgos de personalidad y las dificultades de regulación emocional, con el objetivo de identificar aquellos rasgos que puedan estar implicados en la explicación de las dificultades para regular las emociones que experimentan los estudiantes durante su vida universitaria. Los resultados permitieron mostrar que los rasgos con mayor implicancia sobre las dificultades de regulación emocional fueron Neuroticismo, Extraversión y Amabilidad, siendo mucho más importante el efecto del rasgo Neuroticismo, lo cual es similar a lo reportado por autores como Austin et al. (2008). De manera consistente, la literatura muestra que aquellos individuos que poseen una presencia más marcada de este rasgo suelen presentar mayores dificultades para atender, identificar y reconocer las emociones que están experimentando, así como mayores dificultades para regular sus respuestas emocionales en su exteriorización. Por ejemplo, se ha encontrado que el Neuroticismo permite explicar la desregulación emocional ante situaciones problemáticas y explicar diferencias individuales en las formas de regulación emocional y en el procesamiento de las emociones (e.g., Coffey et al., 2003; Tong, 2010). Tal como plantean Reisenzein y Weber (2009), la relación tan clara entre el Neuroticismo y las emociones se debe a que el Neuroticismo es, por definición, una disposición para la experimentación de emociones negativas.

Respecto de los rasgos Extraversión y Amabilidad, los mismos no resultaron variables predictoras de la forma en la que las personas entienden, identifican y aceptan las emociones, pero sí se asociaron con menores 
dificultades para regular las respuestas emocionales y con la escala considerada en su totalidad. De este modo, aquellas personas con mayor presencia del rasgo Extraversión podrian presentar un mayor nivel de actividad interpersonal $\mathrm{y}$, así, menores dificultades para controlar sus respuestas emocionales, relacionarse positivamente con los demás y reevaluar de manera positiva las situaciones (Augusto Landa et al., 2010; Diener et al., 2003; Kubiak, Wiedig-Allison, Zgoriecki, \& Weber, 2011; Matsumoto, 2006; Reisenzein \& Weber, 2009). Acerca de los resultados hallados para el rasgo Amabilidad, Tobin et al. (2000) indican que las personas con una presencia más marcada del rasgo amabilidad se esfuerzan más por agradar a otros controlando la expresión de las emociones negativas. Sin embargo, cabe mencionar que la incidencia de los rasgos Extraversión y Amabilidad fue menor que la del rasgo Neuroticismo y solo incidió parcialmente sobre las dificultades de regulación emocional.

Asimismo, los rasgos Responsabilidad y Apertura a la Experiencia no resultaron factores predictores de la desregulación emocional. Esto es similar a lo reportado ya en otros estudios (e.g., Augusto Landa et al., 2010), pero difiere de algunas investigaciones que sí reportan relaciones entre estos últimos rasgos y el procesamiento y regulación de las emociones (e.g., Austin et al., 2008; Terracciano et al., 2003). Este resultado nulo podría deberse a que los rasgos Apertura a la experiencia y Responsabilidad tienden a explicar facetas conductuales principalmente individuales (de organización y control de la conducta dirigida a metas), más que aspectos del relacionamiento con los demás (como ocurre con los rasgos Amabilidad y Extraversión). De este modo, dado que la regulación emocional cumple una clara función social, es comprensible que estos rasgos puedan no estar relacionados con la regulación de las emociones. A su vez, tal como plantea Matsumoto (2006), las diferencias halladas en los resultados de los distintos estudios pueden explicarse en parte por aspectos ligados a la cultura. Es decir, que las diferencias para procesar las emociones y poder realizar una gestión exitosa de las mismas puede presentar variaciones en función del patrón cultural y normativo, que las diferentes sociedades establecen sobre cómo, cuándo y en qué medida regular la expresión afectiva.

Los resultados deben ser evaluados a la luz de las limitaciones del estudio. En primer lugar, dadas las características de la muestra, los hallazgos resultan poco generalizables, puesto que solo se trabajó con estudiantes universitarios argentinos, y con una amplia proporción de mujeres respecto de hombres. Otra limitación para considerar es que se trabajó únicamente con cuestionarios de autoinforme, por lo que una posibilidad es que futuros estudios indaguen sobre el tema mediante la utilización de medidas comportamentales o instrumentos más ecológicos. Por último, tampoco fue interés del estudio evaluar estas relaciones en personas con trastornos psicopatológicos. Claramente, con miras a futuras investigaciones, resulta fundamental determinar si estos resultados son replicables en muestras clínicas. En este sentido, y a pesar de las limitaciones, los hallazgos del estudio resultan relevantes dada la importancia de la regulación emocional como eje central en una variedad de sintomas y problemas psicológicos (Vargas Gutiérrez \& Muñoz-Martínez, 2013).

Los estudiantes universitarios son proclives a experimentar dificultades emocionales (Erazo Caicedo \& Jiménez Ruiz, 2012). Por eso, conocer la incidencia de cada rasgo específico de la personalidad sobre la regulación 
emocional en esta población resulta de especial importancia, puesto que los estudiantes son propensos a atravesar situaciones de estrés, malestar y a sufrir síntomas psicopatológicos. Los hallazgos de este estudio sugieren que la presencia de ciertos rasgos de personalidad, en particular Neuroticismo, Amabilidad y Extraversión, permite predecir las posibles dificultades en la regulación emocional que puede presentar un individuo. En particular, el rasgo Neuroticismo presenta el patrón de asociación más claro, tanto a nivel empírico como teórico, siendo mayor la dificultad para reconocer, aceptar e identificar las emociones negativas, así como para regularlas, manejarlas y controlar las respuestas conductuales asociadas a ellas. Este rasgo debe ser considerado en el marco de aquellas intervenciones que tengan por objeto trabajar con las dificultades emocionales en población universitaria.

\section{REFERENCIAS}

Ajzen, I. (2005). Attitudes, personality, and behavior. UK: McGraw-Hill Education.

Allik, J., Realo, A., \& McCrae, R. R. (2013). Universality of the five-factor model of personality. En T. A. Widiger \& P. T. Costa, Jr. (Eds.), Personality disorders and the five-factor model of personality (pp. 6174). American Psychological Association. https://doi.org/10.1037/13939-005

Allport, G. W. (1937). Personality: A psychological interpretation. New York: Holt, Rinehart \& Winston.

American Psychological Association. (2010). Ethical principles of psychologists and code of conduct. Washington, DC: The American Psychologist.

Andrés, M. L. (2014). Efecto mediador de las estrategias cognitivas de regulación emocional en la relación entre los rasgos de personalidad y la ansiedad, depresión y felicidad en niños de 9 a 11 años de edad (tesis doctoral). Universidad Nacional de Mar del Plata, Mar del Plata. Recuperado de http://rpsico.mdp.edu.ar/handle/123456789/538

Andrés, M. L., Canet Juric, L., Castañeiras, C. E., \& Richaud de Minzi, M. C. (2016). Relaciones de la regulación emocional y la personalidad con la ansiedad y depresión en niños. Avances en Psicología Latinoamericana, 34(1), 99-115. https://doi.org/10.12804/apl34.1.2016.07

Andrés, M. L., Castañeiras, C. E., \& Richaud, M. C. (2014). Relaciones entre la personalidad y el bienestar emocional en niños. El rol de la regulación emocional. Cuadernos de Neuropsicología/Panamerican Journal of Neuropsychology, 8(2), 217-241.

Augusto Landa, J. M., Pulido Martos, M., \& López-Zafra, E. (2010). Emotional intelligence and personality traits as predictors of psychological well-being in Spanish undergraduates. Social Behavior and Personality: An International Journal, 38(6), 783-793. https://doi.org/10.2224/sbp.2010.38.6.783

Austin, E. J., Dore, T. C., \& O’Donovan, K. M. (2008). Associations of personality and emotional intelligence with display rule perceptions and emotional labour. Personality and Individual Differences, 44(3), 679688. https://doi.org/10.1016/j.paid.2007.10.001

Carver, C. S. \& Connor-Smith, J. (2010). Personality and coping. Annual Review of Psychology, 61, 679-704. https://doi.org/10.1146/annurev.psych.093008.100352

Cattell, R. B. (1946): The description and measurement of personality. Yonkers-on-Hudson, NY: World.

Coffey, E., Berenbaum, H., \& Kerns, J. (2003). Brief report. Cognition and Emotion, 17(4), 671-679. https://doi.org/10.1080/02699930302304

Costa, P. T. \& McCrae, R. R. (1985). The NEO Personality Inventory manual. Odessa, FL: Psychological Assessment Resources. https://doi.org/10.1037/t07564-000

Côté, G., Gosselin, P., \& Dagenais, I. (2013). Évaluation multidimensionnelle de la régulation des émotions: propriétés psychométriques d'une version francophone du Difficulties in Emotion Regulation Scale. Journal de Thérapie Comportementale et Cognitive, 23(2), 63-72. https://doi.org/10.1016/j.jtcc.2013.01.005

Cupani, M., Garrido, S. J., \& Tavella, J. (2013). El modelo de los cinco factores de personalidad: contribución predictiva al rendimiento académico. Revista de Psicología, 9(17), 67-86.

Diener, E., Oishi, S., \& Lucas, R. E. (2003). Personality, culture, and subjective well-being: Emotional and cognitive evaluations of life. Annual Review of Psychology, 54, 403-425. https://doi.org/10.1146/annurev.psych.54.101601.145056

Erazo Caicedo, M. I., \& Jiménez Ruiz, M. del. C. (2012). Dimensiones psicopatológicas en estudiantes universitarios. Revista CES Psicología, 5(1), 65-76.

Eysenck, H. J. \& Eysenck, M. (1985). Personality and Individual Differences, New York: Plenum Press. https://doi.org/10.1007/978-1-4613-2413-3

Garaigordobil, M., Aliri, J., \& Fontaneda, I. (2009). Bienestar psicológico subjetivo: diferencias de sexo, relaciones con dimensiones de personalidad y variables predictoras. Psicología Conductual, 17(3), 543559.

Giovannini, C., Giromini, L., Bonalume, L., Tagini, A., Lang, M., \& Amadei, G. (2014). The Italian Five Facet Mindfulness Questionnaire: a contribution to its validity and reliability. Journal of Psychopathology and Behavioral Assessment, 36(3), 415-423. https://doi.org/10.1007/s10862-013-9403-0

Goldberg, L. R. (1990). An alternative "description of personality": the big-five factor structure. Journal of Personality and Social Psychology, 59(6), 1216-1229. https://doi.org/10.1037/0022-3514.59.6.1216 
Gratz, K. L. \& Roemer, L. (2004). Multidimensional assessment of emotion regulation and dysregulation: Development, factor structure, and initial validation of the difficulties in emotion regulation scale. Journal of Psychopathology and Behavioral Assessment, 26(1), 41-54. https://doi.org/10.1023/B:JOBA.0000007455.08539.94

Graziano, P. A., Reavis, R. D., Keane, S. P., \& Calkins, S. D. (2007). The role of emotion regulation in children's early academic success. Journal of School Psychology, 45(1), 3-19. https://doi.org/10.1016/j.jsp.2006.09.002

Gross, J. J. (1999). Emotion and emotion regulation. En L. A. Pervin \& O. P. John (Eds.), Handbook of personality: Theory and research (2nd ed.) (pp. 525-552). New York: Guilford Press.

Gumora, G. \& Arsenio, W. F. (2002). Emotionality, emotion regulation, and school performance in middle school children. Journal of School Psychology, 4O(5), 395-413. https://doi.org/10.1016/S00224405(02)00108-5

Guzmán-González, M., Trabucco, C., Urzúa, A., Garrido, L., \& Leiva, J. (2014). Validez y confiabilidad de la versión adaptada al español de la Escala de Dificultades de Regulación Emocional (DERS-E) en población chilena. Terapia Psicológica, 32(1), 19-29. https://doi.org/10.4067/S071848082014000100002

Hervás, G. (2011). Psicopatología de la regulación emocional: el papel de los déficit emocionales en los trastornos clínicos. Psicología conductual, 19(2), 347-372.

Hervás, G. \& Moral, G. (2017). Regulación emocional aplicada al campo clínico. Madrid: Universidad Complutense de Madrid, FOCAD.

Huang, I. C., Lee, J. L., Ketheeswaran, P., Jones, C. M., Revicki, D. A., \& Wu, A. W. (2017). Does personality affect health-related quality of life? A systematic review. PLOS ONE, 12(3), e0173806. https://doi.org/10.1371/journal.pone.0173806

John, O. P., \& Gross, J. J. (2004). Healthy and unhealthy emotion regulation: Personality processes, individual differences, and life span development. Journal of Personality, 72(6), 1301-1334. https://doi.org/10.1111/j.1467-6494.2004.00298.x

Karatzias, T., Shevlin, M., Fyvie, C., Hyland, P., Efthymiadou, E., Wilson, D., ... Cloitre, M. (2016). An initial psychometric assessment of an ICD-11 based measure of PTSD and complex PTSD (ICD-TQ): Evidence of construct validity. Journal of Anxiety Disorders, 44, 73-79. https://doi.org/10.1016/j.janxdis.2016.10.009

Kotov, R., Gamez, W., Schmidt, F., \& Watson, D. (2010). Linking "big" personality traits to anxiety, depressive, and substance use disorders: A meta-analysis. Psychological Bulletin, 136(5), 768-821. https://doi.org/10.1037/a0020327

Kubiak, T., Wiedig-Allison, M., Zgoriecki, S., \& Weber, H. (2011). Habitual goals and strategies in anger regulation. Journal of Individual Differences, 32, 1-13. https://doi.org/10.1027/1614-0001/a000030

Lauretti, P., Villalobos, E., \& González, J. (2007). Programa de Motivación en el Aula: Una Experiencia para el Desarrollo del Docente en Ejercicio. Revista Mexicana de Orientación Educativa, 4(10), 15-22.

Ledesma, R. D., Sánchez, R., \& Diaz-Lázaro, C. M. (2011). Adjective checklist to assess the big five personality factors in the Argentine population. Journal of Personality Assessment, 93(1), 46-55. https://doi.org/10.1080/00223891.2010.513708

Mateo, A. F., Faixa, T. R., \& Martín-Asuero, A. (2014). Mindfulness y regulación emocional: Un estudio piloto. Revista de Psicoterapia, 25(98), 123-132.

Matsumoto, D. (2006). Are cultural differences in emotion regulation mediated by personality traits? Journal of Cross-Cultural Psychology, 37(4), 421-437. https://doi.org/10.1177/0022022106288478

Mauss, I. B., Levenson, R. W., McCarter, L., Wilhelm, F. H., \& Gross, J. J. (2005). The tie that binds? Coherence among emotion experience, behavior, and physiology. Emotion, 5(2), 175-190. https://doi.org/10.1037/1528-3542.5.2.175

McCrae, R. R. \& Costa, P. T. (1987). Validation of the five-factor model of personality across instruments and observers. Journal of Personality and Social Psychology, 52(1), 81-90. https://doi.org/10.1037/0022-3514.52.1.81

McCrae, R. R. \& Costa, P. T. (1991). Adding liebe und arbeit: The full five-factor model and well-being. Personality and Social Psychology Bulletin, 17, 227-232. https://doi.org/10.1177/014616729101700217

McCrae, R. R. \& Costa, P. T. (1997). Personality trait structure as a human universal. American Psychologist, 52(5), 509-516. https://doi.org/10.1037/0003-066X.52.5.509

McLaughlin, K. A., Hatzenbuehler, M. L., Mennin, D. S., \& Nolen-Hoeksema, S. (2011). Emotion dysregulation and adolescent psychopathology: A prospective study. Behaviour Research and Therapy, 49(9), 544-554. https://doi.org/10.1016/j.brat.2011.06.003

Medrano, L. A. \& Trógolo, M. (2014). Validación de la escala de dificultades en la regulación emocional en la población universitaria de Córdoba, Argentina. Universitas Psychologica, 13(4), 1345-1356. https://doi.org/10.11144/Javeriana.UPSY13-4.vedr

Medrano, L. A. \& Trogolo, M. (2016). Construct validity of the Difficulties in Emotion Regulation Scale: further evidence using confirmatory factor analytic approach. Abnormal and Behavioural Psychology, 2(2), 1000117. https://doi.org/10.4172/2472-0496.1000117

Mega, C., Ronconi, L., \& De Beni, R. (2014). What makes a good student? How emotions, self-regulated learning, and motivation contribute to academic achievement. Journal of Educational Psychology, 106(1), 121-131. https://doi.org/10.1037/a0033546

Pérez Díaz, Y. \& Guerra Morales, V. M. (2014). La regulación emocional y su implicación en la salud del adolescente. Revista Cubana de Pediatria, 86(3), 368-375.

Pollock, N. C., McCabe, G. A., Southard, A. C., \& Zeigler-Hill, V. (2016). Pathological personality traits and emotion regulation difficulties. Personality and Individual Differences, 95, 168-177. 
https://doi.org/10.1016/j.paid.2016.02.049

Purnamaningsih, E. H. (2017). Personality and emotion regulation strategies. International Journal of Psychological Research, 10(1), 53-60. https://doi.org/10.21500/20112084.2040

Ramos, F., Morán, C., Pérez, M., \& Manga, D. (2007). Relaciones entre la inteligencia emocional, la personalidad y el cansancio emocional en estudiantes universitarios. Congreso Virtual de Psiquiatría. Sociedad Española de Psiquiatría.

Reisenzein, R. \& Weber, H. (2009). Personality and emotion. En P. J. Corr \& G. Matthews (Eds.), The Cambridge Handbook of personality psychology (pp. 54-71). Cambridge: Cambridge University Press. https://doi.org/10.1017/CBO9780511596544.007

Rodríguez-Camejo, J. (2019). Dos instrumentos comprensivos para la medición de la regulación emocional. Alternativas en Psicologia, 40, 141-155.

Romero, E. (2005). ¿Qué unidades debemos emplear? Las "dos disciplinas" de la psicologia de la personalidad. Anales de Psicología, 21(2), 244-258.

Rusting, C. L. \& Larsen, R. J. (1998). Personality and cognitive processing of affective information. Personality and Social Psychology Bulletin, 24, 200-213. https://doi.org/10.1177/0146167298242008

Tavakol, M. \& Dennick, R. (2011). Making sense of Cronbach's alpha. International Journal of Medical Education, 2, 53. https://doi.org/10.5116/ijme.4dfb.8dfd

Terracciano, A., Merritt, M., Zonderman, A. B., \& Evans, M. K. (2003). Personality traits and sex differences in emotion recognition among African Americans and Caucasians. Annals of the New York Academy of Sciences, 1000, 309-312. https://doi.org/10.1196/annals.1280.032

Tobin, R. M., Graziano, W. G., Vanman, E. J., \& Tassinary, L. G. (2000). Personality, emotional experience, and efforts to control emotions. Journal of Personality and Social Psychology, 79(4), 656-669. https://doi.org/10.1037/0022-3514.79.4.656

Tong, E. M. (2010). Personality influences in appraisal-emotion relationships: The role of neuroticism. Journal of Personality, 78(2), 393-417. https://doi.org/10.1111/j.14676494.2010.00620.x

Vargas Gutiérrez, R. M. \& Muñoz-Martínez, A. M. (2013). La regulación emocional: precisiones y avances conceptuales desde la perspectiva conductual. Psicologia USP, 24(2), 225-240. https://doi.org/10.1590/S0103-65642013000200003

Vélez, L. P. M., Gutiérrez, J. A., \& Isaza, B. E. T. (2010). Depresión en estudiantes universitarios y su asociación con el estrés académico. CES Medicina, 24(1), 7-17.

World Medical Association. (2013). Declaration of Helsinki - Ethical principles for medical research involving human subjects. Recuperado de www.wma.net/policiespost/wma-declaration-of-helsinki-ethicalprinciples-formedical-research-involving-human-subjects /

Recibido 20-02-2020 | Aceptado 26-03-2020

Este trabajo se encuentra bajo una Licencia Creative Commons Atribución 4.0 Internacional que permite a terceros utilizar lo publicado siempre que se dé el crédito pertinente a los autores y a Psicodebate 